\title{
Dynamic Evolution of Information Complexity for Analysis of Design and Development*
}

\author{
Kwang Hee $\mathrm{KO}^{* *}$ Kishore POCHIRAJU*** and Souran MANOOCHEHRI*** \\ ${ }^{* *}$ Departmemt of Mechatronics, Gwangju Institute of Science and Technology \\ 1 Oryong-dong, Bukgu, Gwangju, 500-712, Republic of Korea \\ E-mail:khko@gist.ac.kr \\ ${ }^{* * *}$ Design and Manufacturing Institute and Depatment of mechanical Engineering, \\ Stevens Institute of Technology \\ Hoboken, NJ 07030, USA
}

\begin{abstract}
In this paper we analyze a design and development process of a product or a knowledge-base using complexity and present a methodology to search a sequence using the active complexity, a measure of dynamically changing undefined information. We observe that the information content and parametric interaction of each design process evolve and each design process shows a distinct evolutionary pattern of design information. We analyze such an evolutionary aspect of the design process using complexity theory and optimize it for the minimum active complexity. The obtained optimum design process provides a development sequence with the minimum undefined attributes, which could be used as useful guidance in the product design and assembly planning. We illustrate our approach with examples
\end{abstract}

Key words: Design Process, Complexity, Axiomatic Design, Optimization, Dynamic Complexity, Complexity Evolution

\section{Introduction}

Engineering design may consist of three steps as illustrated in Fig. $1^{(1)}$ : design problem, where the requirements that a final product should fulfill are defined; the design process, which is the methodology used to solve the design problem; and the designed artifact or a system, which is the result of the design process and the final product of the design satisfying the design requirements. These three steps are tightly related, interact with each other, and/or form an iterative loop to reach a design artifact which satisfies all design requirements.

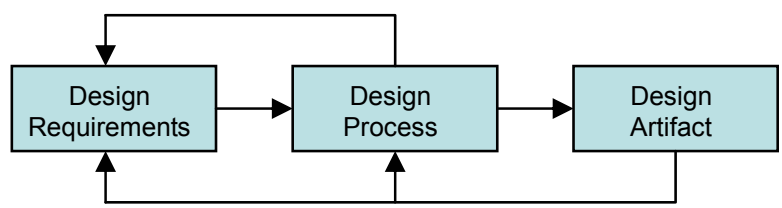

Fig. 1. Overview of design cycle ${ }^{(1)}$

The design process in general consists of multiple steps to reach a design artifact. It can be understood as an iterative process with decomposition and mapping between problem definitions to $\operatorname{artifacts}^{(2)-(4)}$. In other words, the design process is an asynchronous definition

*Received 16 Oct., 2006 (No. 06-0152) [DOI: 10.1299/jamdsm.1.36] 
and/or integration of several elements, i.e. physical geometry, materials, manufacturing processes, assembly, and packaging that meet or exceed the requirement specifications ${ }^{(5)}$.

The design cycle in Fig. 1 may generate more than one design artifact defining the design space, and each design artifact may also be associated with multiple design processes, which define the design process space as shown in Fig. 2.

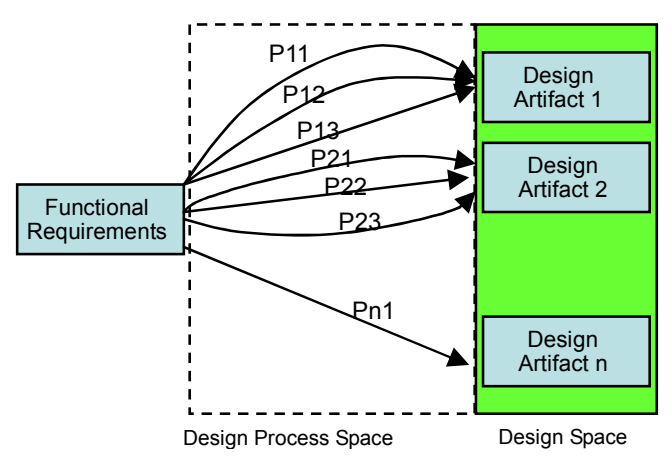

Fig. 2. Concept of design and design process spaces

Suppose that we have a set of design requirements and $n$ design artifacts through $m$ design processes as shown in Fig. 2. Assume that using a certain procedure, the $i$-th artifact has been chosen as our final product. The artifact consists of multiple elements interacting with each other in a complicated manner. Each element does not have to be a physical entity. It could be given as a conceptual one only defining necessary attributes used by other elements. For the $i$-th design artifact, we have multiple design process sequences $\left(P_{i}, P_{i 2}\right.$, $\ldots, P_{i j}$ ) where $1 \leq i \leq n, 1 \leq j \leq r$, and $r$ is the number of design processes to yield the $i$-th design artifact. The design process is evolutionary in that as the process continues, necessary information to define each element will increase and uncertainty will decrease ${ }^{(2)}$. When we resolve uncertainties in all elements, we yield an instance of design, namely, a design artifact. However, during the design process, uncertainty exists and a set of elements needs to be defined. It is obvious that the more uncertainty do we have, the more difficulty do we experience to proceed with the integration of the elements. Therefore, a proper selection of one sequence out of $r$ candidates becomes an important issue in planning product construction or development and minimum uncertainty is a criterion of our choice. In relation to research on the design process, Clarkson and Hamilton ${ }^{(6)}$ proposed a parameter-based model, so called the signposting model, which uses expert knowledge of design tasks and discussed information evolution during the design process. We would like to refer the readers to Refs. (6) and (7) for comprehensive reviews of process design models. Such an evolutionary aspect of design information during the design process can be described by using complexity theory.

Complexity is defined in a variety of ways in different disciplines ${ }^{(1)}{ }^{\text {and }}(8)$. Simon $^{(9)}$ argues that complexity is a property of a system which consists of many parts interacting with each other and whose emergent behavior is hard to understand due to the hierarchical nature of the system. Another popular way to measure complexity is to consider the amount of information that a system may contain ${ }^{(10)}$. This notion of complexity is based on Shannon's information theory and has been used to various applications for measuring complexity of a system. Information theory says that the amount of information does not depend on the particular state of a system, but information is actually contained in the distinction between one state of a system and the other states that the system may have ${ }^{(10)}$. This information can be quantified based on the entropy of information, which is used as a measure of complexity ${ }^{(10)}$. Suh ${ }^{(11)-(13)}$ defines complexity as a measure of uncertainty in achieving a set of functional requirements, which may result from the poor design or insufficient understanding of a system. This measure of complexity relates the amount of information that a designer can access to the probability which the designer can reach a 
valid solution with. It can be reiterated as "the more information is required to achieve the functional requirements of a design, the greater is the complexity of the design". El-Haik and Yang ${ }^{(14)}$ extend the theory by $\operatorname{Suh}^{(11)-(13)}$. Based on this fundamental idea for complexity, many researchers have proposed different forms of complexity in system design and analysis. The analysis of complexity for the design process was performed by several researchers in Refs. (1), (3) and (15)-(19).

The size aspect of a system, such as the number of elements or variables, is directly related with the amount of information contained in the system ${ }^{(5)}$. The more elements are in a system, the greater is the size complexity ${ }^{(10)}$. Although the size itself plays a significant role, how the elements of a system interact with each other also influences the system complexity. Such an interaction is closely related with the links among the elements and can be conceptualized by coupling ${ }^{(1)}$, the density of interactions ${ }^{(20)}$, and the bandwidth ${ }^{(21)}$. These size and link aspects of complexity can describe the complexity of a system and provide a comprehensive picture of the system in terms of complexity ${ }^{(1),(20)}$.

The previous work on the design process analysis based on complexity is mainly focused on the calculation of complexity of the design process for comparison purposes ${ }^{(1)}$. We claim that the analysis of the design process can benefit significantly if we consider an evolutionary complexity of information for analysis of the design process. The complexity evolution can provide more insights of the design process than the simple design information change since it not only captures the change of design information but also reflects how the interactions among elements change. By knowing the information complexity and the parametric dependency at any point during the design process, we can keep track of evolution of complexity. In Ref. (5), we observe that the information contents and parametric interactions of each element for each design process sequence evolve and each design process shows a distinct evolutionary pattern of the size and the link complexity. Such a pattern of the sequence is characterized by interactions among elements involved in the process. Therefore, in this work we propose a method to analyze the sequential aspect of possible design processes from the complexity view point and provide a method to evaluate them for the optimum choice with respect to the minimum uncertainty criterion using complexity.

This paper is structured as follows: in Section 2, the active complexity is defined based on the axiomatic design complexity theory. Section 3 describes the mathematical formulation of the design process optimization, which is followed by implementation of the optimization solution with an XML-based formalized process representation method in Section 4. In Section 5, the proposed method is applied to examples and Section 6 concludes this paper with discussions and future work.

\section{Active Complexity}

In Ref. (5), the size and the link complexity measures are defined and used for the analysis of the structure of a knowledge-base and the evolutionary aspect during product development. They identified the static and dynamic nature of the defined attributes and quantified it using the size and the link complexity measures to describe the change of design information. However, it is noticed that undefined attributes can be directly related with the notion of complexity. Therefore, we consider undefined attributes and their dynamic behaviors in terms of complexity in this paper. Since the previous work in Ref. (5) only measures the size and the link aspects of complexity contributed by defined attributes in each element, we cannot efficiently capture how undefined attributes and links behave during evolution. So in this paper emphasis is placed on the analysis of the evolutionary nature of undefined attributes and we introduce a new definition of complexity for undefined attributes in this section. 


\subsection{Definition}

Consider a system which consists of more than one element. Each element is designed to achieve a set of functional requirements by properly defining a set of attributes, operations, etc. Once all elements are defined, then the system can achieve functional requirements imposed on the system. In this framework, we adopt the axiomatic design complexity definition by $\operatorname{Suh}^{(12)-(13)}$, where the complexity is defined as a measure of uncertainty in achieving a desired set of functional requirements. It is more clearly interpreted by Lee ${ }^{(22)}$; it is a measure of un-likelihood of achieving desired functional requirements. Suh defines the complexity, $I$, as a logarithmic function of the probability of achieving the functional requirements ${ }^{(12)-(13)}$ :

$I=-\log _{2} P_{\{m\}}$,

where $P_{\{m\}}$ is the joint probability that all $m$ functional requirements are satisfied. From such definitions it is derived that complexity is related to information content by arguing that the greater the information required to achieve the functional requirements of a design, the greater is the information content, and thus the complexity ${ }^{(12)}$. Therefore the complexity in this context measures the amount of information necessary to achieve functional requirements of a design. This notion of complexity is consistent with an amount of the undefined information which is needed to completely define the design artifact at each stage of the design process.

During the design process, we need to define each element in a certain order. But the sequence of construction should be governed by inter-relationships between elements. If elements involve no interaction with others, no specific sequence will be preferred and even parallel construction can be performed. When there are interactions between elements, they may drive a certain sequence or an integration order of element definition to reach a design artifact while maintaining uncertainty to a minimum during the design process since some elements are required to be defined in advance in order for a certain element to be defined correctly. This observation motivates the definition of the active complexity. Based on the definition given in Refs. (1) and (10), we define the active complexity as the amount of undefined information (uncertainty) of the elements that have been integrated up to a certain stage of integration of the elements during the design process. Suppose that the amount of undefined information of the $i$-th element at the $k$-th step of an integration sequence is $V_{U i}^{k}$. Then the active complexity at the $k$-th step of the integration order, denoted as $C_{U}^{k}$, is given by

$$
C_{U}^{k}=\sum_{i=1}^{k} \log _{2}\left(V_{U i}^{k}+1\right)
$$

where one is added to $V^{k}{ }_{U i}$ to prevent $C_{U}^{k}$ from having the negative infinity value when there is no undefined information, i.e. $V_{U i .}^{k}=0$.

Depending on the construction sequence, we may compute different active complexity values because interactions between elements are different and a different construction sequence will yield a different pattern of change of information contents. The active complexity has a dynamic nature because during the construction sequence each attribute needs to be defined dynamically which is subject to the current status of other attributes and logical or conditional operations describing each element. Figure 3 illustrates an artifact consisting of three elements with inter-elemental references. The attribute $a$ in Element 1 will be defined only if both $c$ in Element 3 and $b$ in Element 2 are defined. This structure indicates that starting with Element 1 will contain an undefined attribute, leading to high active complexity during development process. On the other hand, if we define Element 3 first and proceed with Element 2 and Element 1, then we keep the active complexity to a minimum. 


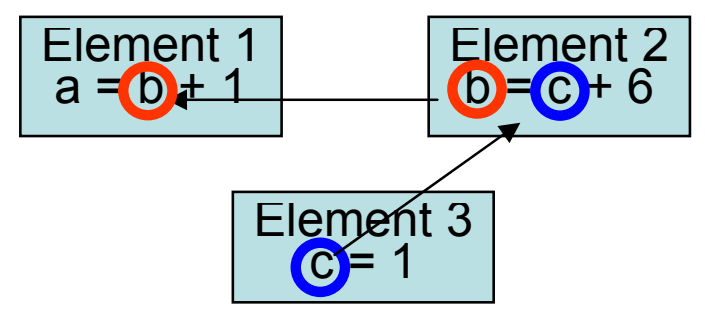

Fig. 3. Schematic of three elements with interactions

This example illustrates that a particular order of development will maintain the active complexity to a minimum. Following this order, we can minimize uncertainty in defining each element, leading to an optimum development sequence in terms of minimum information complexity. Searching such a development order is the main problem of this paper.

\subsection{Relation with Other Complexity Measures}

The static and the dynamic complexity measures are introduced in Ref. (5). In this section, we investigate a relation between the active complexity, and the static and the dynamic complexity.

The static complexity describes the structure of a given design. Namely, once a design is done, the overall structure, the size and dependency between attributes and elements, is determined $^{(5)}$. Suppose that we have an artifact represented with $E$ number of elements. We denote the static complexity at the $k$-th step in the development sequence as $C_{s}^{k}$, which is given as follows ${ }^{(5)}$ :

$$
C_{s}^{k}=\sum_{i=1}^{k} \log _{2}\left(V_{i}+1\right),
$$

where $V_{i}$ is the total number of attributes used in the $i$-th element. The dynamic complexity depends on the dependency between attributes and elements. Therefore, depending on the development sequence, the defined attributes will dynamically change. Such a change may not be easily revealed from the structure alone. Instead, we need to trace it at each development step. Once all elements are integrated, then the dynamic complexity should be equal to the static complexity. Suppose that the number of defined attributes in the $i$-th element is denoted as $V_{D i}^{k}$ at the $k$-th step of a development sequence. Then the dynamic complexity for size at the $k$-th step, $C_{D}^{k}$, is given as:

$$
C_{D}^{k}=\sum_{i=1}^{k} \log _{2}\left(V_{D i}^{k}+1\right) \text {. }
$$

When $k=E$, then the dynamic complexity is equal to the static complexity. Suppose we denote the number of undefined elements in the $i$-th element as $V^{k}{ }_{U i}$. It is obvious that we have a relation between the three numbers $V_{i}, V_{D}^{k}$, and $V_{U i}^{k}, V_{i}=V_{D i}^{k}+V_{U i}^{k}$. Then,

$$
\begin{aligned}
C_{s}^{k}-C_{D}^{k} & =\sum_{i=1}^{k} \log _{2}\left(V_{i}+1\right)-\sum_{i=1}^{k} \log _{2}\left(V_{D i}^{k}+1\right)=\sum_{i=1}^{k} \log _{2}\left(\frac{V_{i}+1}{V_{D i}^{k}+1}\right)=\sum_{i=1}^{k} \log _{2}\left(\frac{V_{i}+1}{V_{i}-V_{U i}^{k}+1}\right) \\
& =\sum_{i=1}^{k} \log _{2}\left(\frac{1}{1-\frac{V_{U i}^{k}}{V_{i}+1}}\right)=\sum_{i=1}^{k} \log _{2}\left(\frac{1}{1-\gamma_{i}}\right) .
\end{aligned}
$$

Here

$\gamma_{i}=\frac{V_{U i}^{k}}{V_{i}+1}$

is the ratio of the undefined attributes to the total number of attributes. When there is no $V_{U i}^{k}$ 
for all $1 \leq i \leq k$, then $\gamma_{i}=0$ and $C_{s}^{k}=C_{D}^{k}$, which is the ideal case in the product development. The ratio $\gamma_{i}$ is proportional to $V_{U i}^{k}$ and in turn is related to $C_{U}^{k}$. Therefore, $C_{s}^{k}-C_{D}^{k}$ is directly related with the active complexity $C_{U}^{k}$.

\section{Problem Formulation}

In this section, we present a mathematical formulation of searching an optimum design process. The active complexity is used in this formulation as a measure for optimization. Since the amount of undefined information becomes the direct measure of the active complexity as in Eq. (2), for the sake of computational efficiency, we use the amount of undefined information without computing the active complexity.

\subsection{Formulation}

Suppose $\boldsymbol{S}$ is a set of $n$ elements, $\boldsymbol{S}=\left\{s_{1}, s_{2}, \ldots, s_{n}\right\}$. We define $\boldsymbol{P}$ as the set of $n$ ! permutations of $\boldsymbol{S}$ and $p$ is one instance of $\boldsymbol{P}$, which indicates one sequence of the $n$ elements. For example, $p$ would be $p=s_{2} s_{3} s_{n} \ldots s_{1}$. Given $p$, we define $\Gamma_{k}^{p}$ to be a sub-sequence of $p$, which contains from the left most of $p$ up to the $k$-th element in $p$. A function, $f\left(\Gamma_{k}^{p}\right)$, computes a certain characteristic value when a process in $\Gamma_{k}^{p}$ sequence is processed. Then, the optimization problem is to find one $p$ such that

$$
\Phi=\sum_{k=1}^{n} f\left(\Gamma_{k}^{p}\right)^{2}
$$

becomes minimal.

In this formulation, the set $\mathbf{S}$ represents a design artifact consisting of $n$ elements. A permutation $p$ indicates a sequence of construction or a design process to produce the artifact and $\Gamma_{k}^{p}$ is a partial artifact which has been constructed using the first $k$ elements in $p$. The function $f\left(\Gamma_{k}^{p}\right)$ computes the amount of undefined information of the partial artifact containing the first $k$ elements in $p$.

\subsection{Solution}

As the number of elements and the dependency patterns get more and more complex, then the manual trace of the active complexity becomes impossible. In addition, nested dependency between attributes may make the analysis intractable. Therefore, in order to solve Eq. (7) we need a systematic optimization tool.

The problem described in Section 3.1 is characterized as a combinatorial optimization, i.e. given a possible set of instances, we search one which optimizes the formulated problem. There may be a few approaches to attack this problem and in this paper we employ the simulated annealing method ${ }^{(23)-(24)}$, which is widely accepted as an efficient solution method for combinatorial optimization problems.

\section{Implementation}

In this work, we use the same method as in the authors' previous work ${ }^{(5)}$ to represent an artifact or a system. For the representation of attributes and functions in each element, we use the XML based frame-slot structure. We also use an efficient algorithm to handle dynamic change of attributes in each element explained in Ref. (25). In this section, we briefly introduce the method presented in Ref. (5) and describe the overall software structure for solving the problem given in Section 3.

\subsection{Representation}

The frame structure was originally proposed to represent a knowledge set ${ }^{(26)}$. In this paper, we use the similar representation scheme to describe the internal structure of each 
element using XML ${ }^{(5)}$. The XML tags used in representation are summarized in Table 1. The attribute of a frame has a functional form. The left-hand-side of the attribute is defined in the tag $<$ LHS $><$ LHS $>$. The right-hand-side, given in $<$ RHS $></$ RHS $>$, whose results are assigned to the LHS, can be algebraic expressions, logical, relational, symbolic or list expressions. Various numerical as well as non-numerical attributes including symbols, geometric shapes and logical values can be provided in the tag. For details, we would like to refer to Ref. (5).

\begin{tabular}{|l|l|}
\hline \multicolumn{1}{|c|}{ Tags } & \multicolumn{1}{|c|}{ Descriptions } \\
\hline$<$ system $></$ system $>$ & Definition of system \\
\hline$<$ frame $></$ frame $>$ & Definition of frame \\
\hline$<$ name $></$ name $>$ & Definition of frame name \\
\hline$<$ input $></$ input $>$ & Definitions of each section \\
\hline$<$ output $></$ output $>$ & \\
\hline$<$ formulae $></$ formulae $>$ & Control of behavior \\
\hline$<$ control $></$ control $>$ & Definition of complexity \\
\hline$<$ complexity $></$ complexity $>$ & Definition of attribute \\
\hline$<$ attribute $></$ attribute $>$ & Left-hand-side \\
\hline$<$ LHS $></$ LHS $>$ & Right-hand-side \\
\hline$<$ RHS $></$ RHS $>$ & ner-defined $x M L$ \\
\hline
\end{tabular}

Table 1. Descriptions of user-defined XML tags ${ }^{(5)}$.

A frame defined in XML is interpreted by a knowledge processing unit ${ }^{(25)}$ to generate a tree. It has a root node having section nodes as children. Each section node contains attributes which are also represented as trees. Dependency between attributes is made by using attributes' name. There exist two kinds of dependency in the frame-base representation: within a frame and across frames. An attribute defined as a slot in a frame can reference any other attribute within the same frame by just calling its name, i.e. slot. To reference an attribute in other frame both the frame and the attribute names must be specified in the following format, :frame_name.attribute_name. Dependency tracking and cyclic component detection are employed in the processing unit. Through dependency tracking, a change in an attribute propagates through a dependency chain created during frame interpretation and all the attributes involved in the dependency track are updated. The dependency chain is also used to detect a cycle. When a loop is detected, an error message is generated to warn the user of the existence of a cycle. This capability is critical to prevent the propagation of change from being trapped in an infinite loop.

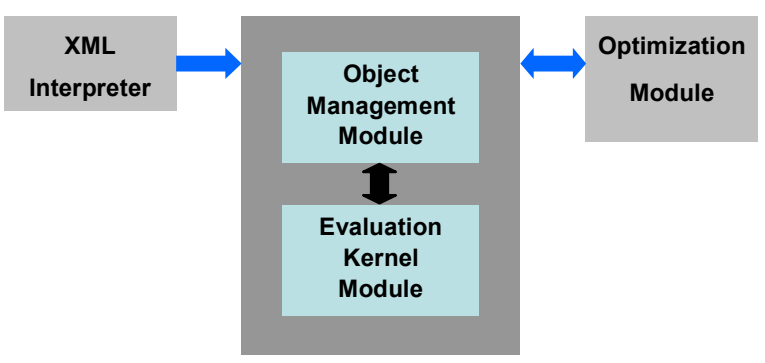

Fig. 4. Schematic of Software Structure

\subsection{Software Implementation}

The software consists of three major units as shown in Fig. 4. The XML interpreter module receives input XML data, parses and stores them in a tree data structure. From this parsed data, an object for each element is created by the object management module, which also maintains each object status, updates changes made to an object and interacts with the evaluation kernel module. The evaluation kernel module performs expression interpretations and evaluations, referencing and tracking. It is designed to handle various 
forms of attributes including non-numerical values. The optimization module interacts with the object management module. For optimization, we use the simulated annealing $\operatorname{method}^{(23)}$, which is suitable for combinatorial optimization problems.

\section{Applications}

In this section we demonstrate our approach with examples and discuss the results of each case. We create a design artifact consisting of multiple elements each of which contains arbitrary attributes and interactions among elements, and then analyze it using the active complexity measure. Then we take a real working knowledge-base example developed for injection molding for analysis.

\subsection{Analysis of Design Artifact}

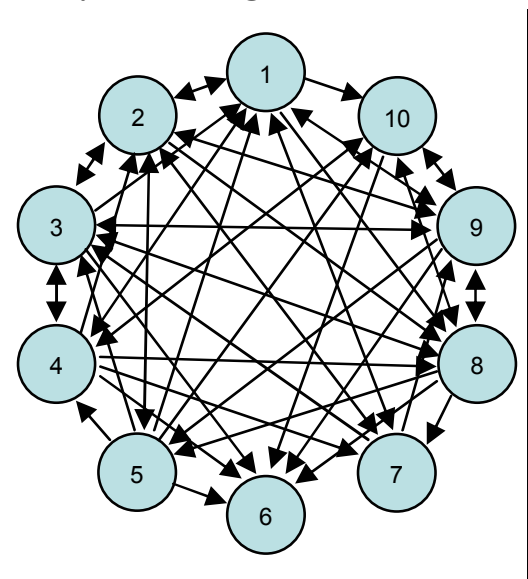

Fig. 5. Schematic of element interactions

\begin{tabular}{|c|c|c|c|c|c|c|c|c|c|c|c|}
\hline No. & 1 & 2 & 3 & 4 & 5 & 6 & 7 & 8 & 9 & 10 & Sum \\
\hline 1 & $\mathrm{X}$ & 2 & 0 & 1 & 0 & 0 & 1 & 1 & 1 & 1 & 7 \\
\hline 2 & 3 & $\mathrm{X}$ & 1 & 0 & 1 & 0 & 1 & 1 & 1 & 0 & 8 \\
\hline 3 & 1 & 1 & $\mathrm{X}$ & 1 & 0 & 1 & 0 & 1 & 1 & 0 & 6 \\
\hline 4 & 1 & 1 & 1 & $\mathrm{X}$ & 0 & 1 & 1 & 1 & 0 & 2 & 8 \\
\hline 5 & 2 & 1 & 2 & 1 & $\mathrm{X}$ & 1 & 0 & 0 & 0 & 1 & 8 \\
\hline 6 & 0 & 0 & 0 & 0 & 0 & $\mathrm{X}$ & 0 & 0 & 0 & 0 & 0 \\
\hline 7 & 5 & 1 & 1 & 0 & 0 & 0 & $\mathrm{X}$ & 0 & 1 & 0 & 8 \\
\hline 8 & 0 & 0 & 1 & 0 & 3 & 2 & 1 & $\mathrm{X}$ & 1 & 1 & 9 \\
\hline 9 & 2 & 1 & 1 & 0 & 1 & 1 & 0 & 1 & $\mathrm{X}$ & 2 & 9 \\
\hline 10 & 0 & 0 & 0 & 2 & 0 & 1 & 0 & 1 & 1 & $\mathrm{X}$ & 5 \\
\hline Sum & 14 & 7 & 7 & 5 & 5 & 7 & 4 & 6 & 6 & 7 & 68 \\
\hline
\end{tabular}

Table 2. Number of external references for each element

We first test our proposed approach with an example of a fictitious design artifact consisting of ten elements or frames that are referencing to each other in a fairly complex way as illustrated in Fig. 5. Each element is assigned a unique number from one to ten for unique identification. .

The total number of external references between elements is 68 , which is summarized in Table 2. There exist nested references inside each element but they are not shown in the table and the figure. The elements in columns are referenced by the elements in rows and the letter ' $\mathrm{X}$ ' means no external reference exists.

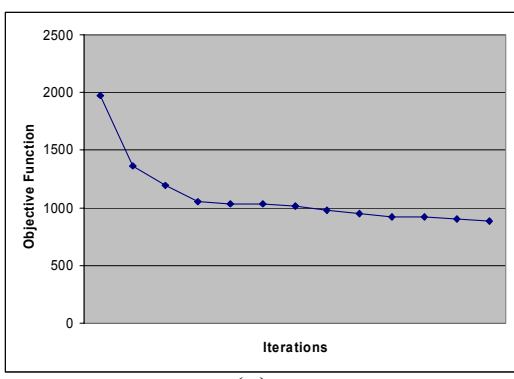

(a)

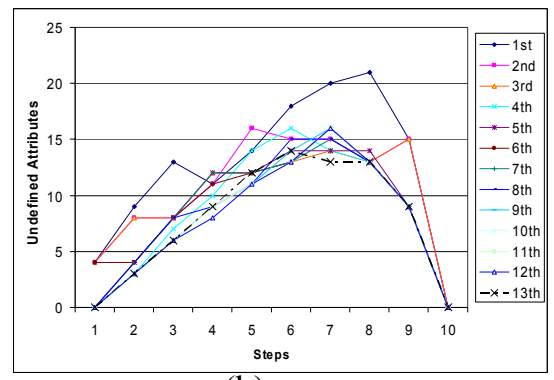

(b)

Fig. 6. (a) Change of objective function values during optimization (b) Evolution of undefined attributes for each development sequence.

The initial order of the development sequence is given randomly. Figure 6 (a) shows the change of the objective function values during optimization. After the optimization, we obtain the most probable global optimum sequence, $(6,8,7,1,2,3,4,10,9,5)$ with the objective function value, 885. Figure 6 (b) shows how the number of undefined attributes evolves for each selected development sequence. The optimum sequence in the design process indicates 
that when an artifact is developed, the attributes in Element 6 should be first defined in order for the attributes of the subsequent elements to be defined with the maximum amount of known information.

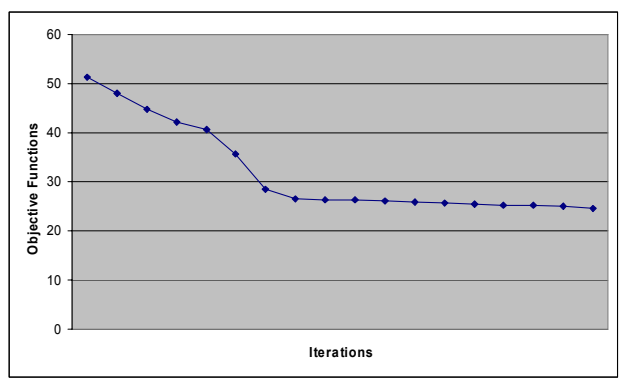

Fig. 7. Change of objective function values during optimization

We solve the same problem by optimizing $C_{s}^{k}-C_{D}^{k}$ instead of computing the amount of undefined information, $V_{U i}^{k}$, in Eq. (2). The change of the objective function values is given in Fig. 7. The optimum value is 24.572 , whose corresponding sequence is the same as the one obtained in Fig. 6 (a). As shown in Section 2.2, optimizing either $C_{S}^{k}-C_{D}^{k}$ or $V_{U i}^{k}$ reaches the same answer.

\subsection{Analysis of Knowledge-base}

A knowledge-base in general consists of domain specific knowledge sets, each of which is defined with models, rules, constraints and parameters and interacts with others. Each knowledge set is treated as an element and the aggregate of all elements, namely, the fully developed knowledge-base as a design artifact. At each design process step, related domain knowledge sets should be defined and integrated to form a complete knowledge-base. So it can be easily understood that there exist more than one design processes to reach the knowledge-base. In this section, we take an already developed knowledge-base ${ }^{(5)}$ as a design artifact and analyze it using the proposed method in this paper.

\subsubsection{Knowledge-base Representation}

We employ the frame-slot architecture presented in Section 4.1 to store the parameters, arithmetic expressions and mathematical functions as well as constraints describing design. This frame-slot architecture is a good way to represent a design knowledge-base since under this architecture engineering design knowledge can be easily formalized and stored as mathematical expressions, functions and a set of constraints.

\subsubsection{Analysis of Knowledge-base for Injection Molding Process}

The knowledge-base describes five domain specific elements, each of which entails specification of materials, tools, geometry, process and cost economics ${ }^{(5)}$. Figure 8 shows a schematic diagram of the five elements of the knowledge-base. The arrows depict the data dependencies between elements. Properties of a material of the product are defined in 'Material' element and 'Tool' element contains parameters of the injection machine considered in the injection molding process. The surface area and volume of a product which are critical in estimation of cost are computed in 'Geometry' element. 'Process' element contains a knowledge set on the injection molding manufacturing process such as the part production cycle time and required machine capacity. The various costs such as the mold design cost, production cost, etc. are stored in 'Economics' element.

The parametric dependencies between the design elements are summarized in Table 3. In Ref. (5), the same knowledge-base was analyzed in terms of the static, the dynamic size and the link complexity, which capture how design information grows as the design process proceeds. However, in this work, we focus on each design process based on the evolution of 
the active complexity to search an optimum process.

As in Ref. (5) we consider three possible sequences for the process steps in order to evaluate complexity evolution during the design process. The first sequence is the geometry-first one in which geometry is defined first, and then the material and the process are defined. The second process considered is the material-first approach in which the material and the process are defined before the geometry and the last is the process-first approach in which the process is defined first followed by the material and the geometry. Table 4 shows the sequence of steps for each of the process sequences ${ }^{(5)}$.

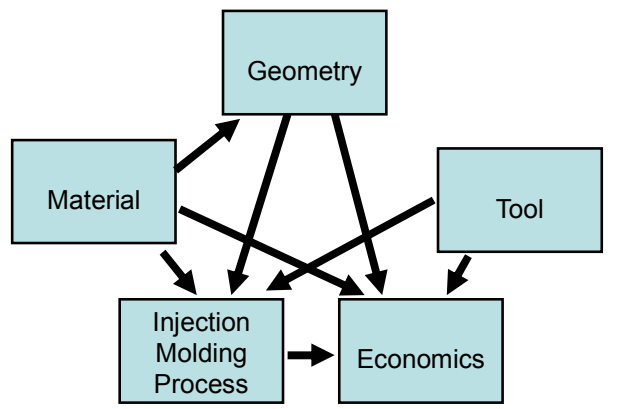

Fig. 8. Schematic of the elements of the knowledge-base and their dependencies(5).

\begin{tabular}{|c|c|c|c|c|c|}
\hline & Geometry & Process & Tool & Material & Economics \\
\hline Geometry & $\mathrm{X}$ & 0 & 0 & 1 & 0 \\
\hline Process & 8 & $\mathrm{X}$ & 7 & 22 & 0 \\
\hline Tool & 0 & 0 & $\mathrm{X}$ & 0 & 0 \\
\hline Material & 0 & 0 & 0 & $\mathrm{X}$ & 0 \\
\hline Economics & 13 & 8 & 4 & 2 & $\mathrm{X}$ \\
\hline
\end{tabular}

Table 3. External dependencies between various design elements ${ }^{(5)}$

We may consider alternative design process sequences such as the economy-first approach. However, the sequences considered in this example are three commonly used ones in practice. So we limit our problem domain to those three candidates.

\begin{tabular}{|c|c|c|c|c|c|c|c|c|c|}
\hline Step & \multicolumn{3}{|c|}{ Geometry First } & \multicolumn{4}{c|}{ Material First } & \multicolumn{3}{c|}{ Process First } \\
\hline & & $\mathrm{UL}$ & $\mathrm{C}_{\mathrm{u}}$ & & $\mathrm{UL}$ & $\mathrm{C}_{\mathrm{u}}$ & & $\mathrm{UL}$ & $\mathrm{C}_{\mathrm{u}}$ \\
\hline 1 & $\mathrm{G}$ & 3 & 2 & $\mathrm{M}$ & 0 & 0 & $\mathrm{P}$ & 60 & 5.93 \\
\hline 2 & $\mathrm{M}$ & 0 & 0 & $\mathrm{P}$ & 35 & 5.17 & $\mathrm{M}$ & 35 & 5.17 \\
\hline 3 & $\mathrm{P}$ & 9 & 3.32 & $\mathrm{G}$ & 9 & 3.32 & $\mathrm{G}$ & 9 & 3.32 \\
\hline 4 & $\mathrm{~T}$ & 0 & 0 & $\mathrm{~T}$ & 0 & 0 & $\mathrm{~T}$ & 0 & 0 \\
\hline 5 & $\mathrm{E}$ & 0 & 0 & $\mathrm{E}$ & 0 & 0 & $\mathrm{E}$ & 0 & 0 \\
\hline
\end{tabular}

Table 4. Three design process sequences, number of undefined attributes in the knowledge-base and the active complexity. (G : Geometry, M : Material, P : Process, T : Tool, E : Economics, UL : Undefined Links and $\mathrm{C}_{\mathrm{u}}$ : Active Complexity)

Figure 9 shows how the active complexity evolves depending on each design process sequence. The vertical axis is the active complexity and the horizontal axis is the step number given in Table 4.

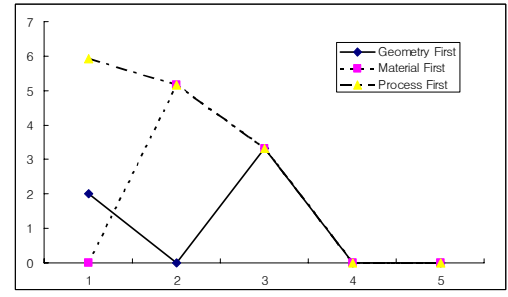

Fig. 9. Evolution of active complexity for each design process sequence. 
While the process-first sequence has monotonically decreasing number of undefined variables through the sequence, the material-first sequence has peak after the definition of the material and the process but without a definition of geometry. We find that the optimum sequence is to start with defining geometry, and subsequently to define material, process, tool and economics elements. It is implied that with a definition of the geometry of a product, the subsequent elements can be properly defined with the maximum amount of information, namely, the minimum amount of undefined attributes.

\section{Concluding Remarks}

In this paper, we present the concept of the active complexity to describe the dynamic behavior of design information of an artifact during the design process. The active complexity quantifies the amount of undefined attributes at each development step, which is a property inherent to each development sequence. Using this complexity measure, we formulate an optimization problem to search one sequence which yields the minimum active complexity through the simulated annealing process. The sequence with the optimum active complexity bears the minimum amount of undefined information at each development step, which may lead to the optimum development process plan of a design artifact.

The active complexity will give a novel perspective of how each element is interacting with others and how design information evolves during development, which can provide a different way of analyzing the design process. Analysis of design sequence process is beneficial in that in planning a process of a real product, the product can be developed with maximum design information at each step by following the optimum development process, which can be used for efficient time management for product development, resource allocation and optimum product development schedule development. It is demonstrated that the information complexity can be served as a useful tool in the analysis of a product development process and in addition be used as a driver for the optimum choice of a design process as opposed to the process selection of minimum cost. As future work it is recommended that the relation between complexity and other parameters such as cost and time be sought mathematically.

\section{Acknowledgement}

The authors acknowledge with appreciation the support provided by US/ARMY ARDEC under the contract DAAE30-97-C-1041 for Systems Engineering Research, FY 2004.

\section{References}

(1) Summers, J. D., and Shah, J. J., Developing Measures of Complexity For Engineering, Design, Proceedings of DETC'03 ASME 2003 Design Engineering Technical Conferences and Computers and Information in Engineering Conference, (2003).

(2) Braha, D., and Maimon, O., The Design Process: Properties, Paradigms, and Structure, IEEE Transactions on Systems, Man and Cybernetics-Part A: Systems and Humans, Vol. 27, (1997), pp. 146-166.

(3) Braha, D., and Maimon, O., The Measurement of a Design Structural and Functional Complexity, IEEE Transactions on Systems, Man and Cybernetics-Part A: Systems and Humans, Vol. 28, (1998), pp. 527-535.

(4) Hazelrigg, G., Systems Engineering: An Approach to Information-Based Design, (1996) Prentice Hall

(5) Ko, K. H., et al., Analysis of Information Complexity During Product Development, Proceedings of IDETC/CIE 2005, ASME 2005 International Design Engineering Technical Conferences \& Computers and Information in Engineering Conference, 
(2005).

(6) Clarkson, P. J., and Hamilton, J. R., 'Signposting', A Parameter-driven Task-based Model of the Design Process, Research in Engineering Design, Vol. 12, (2000), pp. 18-38.

(7) Ullman D. G., The Mechanical Design Process, (1997), McGraw Hill.

(8) Sussman, J. M., Ideas on Complexity in Systems - Twenty Views, Working paper series, ESD-WP-2000-02, (http://esd.mit.edu/wps/), , (2000), Massachusetts Institute of Technology Engineering Systems Division.

(9) Simon, H. A., The Sciences of the Artificial, (1998),Cambridge.

(10) Bar-Yam, Y., Dynamics of Complex Systems, (1997), Perseus Books.

(11) Suh, N., A Theory of Complexity, Periodicity, and the Design Axioms, Research in Engineering Design, Vol. 11, (1999), pp. 116-31.

(12) Suh, N. P., Axiomatic Design: Advances and Applications, (2001), Oxford University Press.

(13) Suh, N. P., Complexity Theory and Applications, (2005), Oxford University Press.

(14) El-Haik, B., and Yang, K., The Components of Complexity in Engineering Design, IIE Transactions, Vol. 31, (1999), pp. 925-34.

(15) Ahn, J., and Crawford, R., Complexity Analysis of Computational Engineering Design Processes, DTM '94, ASME, Design Theory and Methodology, Vol. 68, (1994), pp. 205-220.

(16) Gammerman, A., and Vovk, V., Kolmogorov Complexity: Sources, Theory and Applications, The Computer Journal, Vol. 42, (1999), pp. 252-255.

(17) McCabe, T., and Butler, C., Design Complexity Measurement and Testing, Communications of the ACM, Vol. 32, (1989), pp. 1415-1425.

(18) Varma, D., and Trachtenberg, E., On the Estimation of Logic Complexity for Design Automation Applications, IEEE International Conference on Computer Design: VLSI in Computers and Processors, (1990).

(19) Earl, C. F., and Eckert, C. M., Complexity of Planning in Design, Proceedings of DETC' 01, 13th International Conference on Design Theory and Methodology, (2001).

(20) Martin, P.-A. J. Y., A Framework for Quantifying Complexity and Understanding its Sources: Application to two Large-Scale Systems, MS Thesis, (2004), Massachusetts Institute of Technology.

(21) Tang, V., and Salminen, V., Towards a Theory of Complicatedness: Framework for Complex Systems Analysis and Design, 13th International Conference on Engineering Design, (2001).

(22) Lee, T., Complexity Theory in Axiomatic Design, PhD Thesis, (2003), Massachusetts Institute of Technology.

(23) Kirkpatrick, S., Gelatt Jr., C. D., and Vecchi, M. P., Optimization by Simulated Annealing, Science, Vol. 220, (1983), pp. 671-680.

(24) Nahar, S., Sahni, S., and Shragowitz, E., Simulated Annealing and Combinatorial Optimization, Proceedings of the 23rd ACM/IEEE conference on Design automation, (1986), pp. $293-299$.

(25) ACES 3.0, Technical Report, Enhancement of ACES for Ammunitions Applications, (2004), Design and Manufacturing Institute, Stevens Institute of Technology.

(26) Minsky, M., A Framework for Representing Knowledge, The Psychology of Computer Vision, edited by P. H. Winston, (1975), McGraw-Hill Book. 